\title{
Speech structure links the neural and socio-behavioural correlates of psychotic disorders
}

\author{
Lena Palaniyappan $^{\mathrm{a}, *, 1}$, Natália Bezerra Mota ${ }^{\mathrm{b}, \mathrm{c}, 1}$, Shamuz Oowise $^{\mathrm{d}}$, Vijender Balain ${ }^{\mathrm{e}}$, \\ Mauro Copelli ${ }^{\mathrm{c}}$, Sidarta Ribeiro ${ }^{\mathrm{b}}$, Peter F. Liddle ${ }^{\mathrm{f}, \mathrm{g}}$ \\ a Department of Psychiatry, Robarts Research Institute, University of Western Ontario, Lawson Health Research Institute, London, ON, Canada \\ ${ }^{\mathrm{b}}$ Brain Institute, Federal University of Rio Grande do Norte (UFRN), Natal, Brazil \\ ${ }^{c}$ Physics Department, Federal University of Pernambuco (UFPE), Recife, Brazil \\ d The Forensic and Long Bay Hospitals, Justice and Forensic Mental Health Network, Sydney, NSW, Australia \\ e Penticton Regional Hospital, 550 Carmi Avenue, Penticton, British Columbia, Canada \\ ${ }^{\mathrm{f}}$ Centre for Translational Neuroimaging, Institute of Mental Health, Nottingham, UK \\ ${ }^{g}$ Division of Psychiatry \& Applied Psychology, University of Nottingham, Nottingham, UK
}

\section{A R T I C L E I N F O}

\section{Keywords:}

Disorganization

Bipolar disorder

Schizophrenia

Thought disorder

Gyrification

Centrality

Graph theory

\begin{abstract}
A B S T R A C T
Background: A longstanding notion in the concept of psychosis is the prominence of loosened associative links in thought processes. Assessment of such subtle aspects of thought disorders has proved to be a challenging task in clinical practice and to date no surrogate markers exist that can reliably track the physiological effects of treatments that could reduce thought disorders. Recently, automated speech graph analysis has emerged as a promising means to reliably quantify structural speech disorganization. Methods: Using structural and functional imaging, we investigated the neural basis and the functional relevance of the structural connectedness of speech samples obtained from 56 patients with psychosis (22 with bipolar disorder, 34 with schizophrenia). Speech structure was assessed by non-semantic graph analysis. Results: We found a canonical correlation linking speech connectedness and i) functional as well as developmentally relevant structural brain markers (degree centrality from resting state functional imaging and cortical gyrification index) ii) psychometric evaluation of thought disorder iii) aspects of cognitive performance (processing speed deficits) and iv) functional outcome in patients. Of various clinical metrics, only speech connectedness was correlated with biological markers. Speech connectedness filled the dynamic range of responses better than psychometric measurements of thought disorder. Conclusions: The results provide novel evidence that speech dysconnectivity could emerge from neurodevelopmental deficits and associated dysconnectivity in psychosis.
\end{abstract}

\section{Introduction}

Psychotic disorders such as schizophrenia were originally conceptualized as conditions exhibiting loosening of associations in thought processes (Bleuler, 1950) and a weakening of associative-links in the brain (Lanczik and Keil, 1991). Loosening of associations in thought processes manifest as speech disturbances in patients. Neuroimaging investigations, on the other hand, provide the means to quantify brain dysconnectivity in psychosis. Understanding the relationship between these two levels of dysconnectivity is likely to provide an important lead in the pathophysiology of psychosis (Goghari et al., 2010). Although several attempts have been made to ascertain this connection (Sumner et al., 2017; Cavelti et al., 2018; Kircher et al.,
2018), consistent neurobiological features underpinning thought disorder in psychosis are yet to be identified.

Assessment of the subtle aspects of thought disorders is a challenging task in clinical practice (Chapman and Chapman, 1973). Semistructured instruments are often insufficient to detect subtle speech deficits during the course of a clinical interview. The use of speech samples (e.g. Thought and Language Index (Liddle et al., 2002a)) has improved the detection rate to some extent, making it possible to detect deviations in speech even in apparently healthy individuals (Sommer et al., 2010), though clinical judgment plays a key role in identifying such deficits. This introduces significant inter-rater variability (Liddle et al., 2002a), hindering efforts to understand the brain-symptom relationship. Recently, a novel approach using non-semantic graph

\footnotetext{
* Corresponding author at: Room 1232D, Robarts Research Institute, 100 Perth Drive, London, ON N6A 5K8, Canada.

E-mail address: lpalaniy@uwo.ca (L. Palaniyappan).

${ }^{1}$ Equivalent contribution.
} 
analysis to calculate the connectedness between words has been suggested to quantify the manifest-scale dysconnectivity in speech samples (Mota et al., 2017; Mota et al., 2014; Mota et al., 2012). Specifically, it was shown that patients with schizophrenia diagnosis report their memories with lower connectedness between words, in comparison with non-psychotic individuals, or patients with psychotic symptoms diagnosed with bipolar disorder (Mota et al., 2017; Mota et al., 2014; Mota et al., 2012). One advantage of this new approach is to be naturalistic, analysing the free discourse of the patient, as manifested in the clinical interview. Second, it is completely independent of the subjective interpretation of symptoms. Third, this method allows for systematic comparison using multiple random graphs made with the same words used by the patient, thus improving the measurement precision (Mota et al., 2017). Thus, speech graph analysis might relate readily to the elusive pathophysiology of disorganization or formal thought disorder (FTD) seen in psychosis.

FTD is a feature of various psychotic disorders, including schizophrenia and bipolar disorder (Passby and Broome, 2017), with some elements of speech disturbances being observed more often in one disorder than the other (Andreasen and Grove, 1986; Hoffman et al., 1986). A valid measure of thought disorder can be expected to detect thought disturbances and its severity irrespective of diagnostic boundaries (i.e. schizophrenia and bipolar disorder with psychosis), while at the same time contributing to the clinical distinction among the disorders. Therefore, we expected to replicate previous results and find lower connectedness between words in patients with the schizophrenia diagnosis, in comparison with patients with psychotic symptoms and a diagnosis of bipolar disorder. Given the importance of FTD in defining the nature of psychosis and predicting its clinical (Demjaha et al., 2017) and global functional impact (Cavelti et al., 2016), one must be able to relate such a measure to functional outcome as well as any existing scales for FTD. The measure should also be expected to relate to core cognitive deficits in psychoses such as processing speed (Dickinson et al., 2007), as FTD shows a complex interplay with cognitive impairment (Xu et al., 2014; Nagels et al., 2016). Finally, this measure can also be expected to relate to functional and structural measures of neuroanatomical measures.

Neuroimaging allows several variables reflecting brain dysconnectivity to be derived from subjects with psychosis. Disruption in connectivity during early development results in abnormalities in cortical folding and surface area. Unlike DTI that provides a current index of structural connectivity, the degree of cortical folding (gyrification) (White and Hilgetag, 2011; Dubois et al., 2008) provides a quantitative proxy of the degree of developmental brain connectivity in subjects with psychosis. Degree centrality, a measure based on the application of graph theory to brain networks, captures the number of functional connections between a single voxel and the rest of the brain (Buckner et al., 2009). Certain brain regions have a high degree centrality and act as core hubs for connectivity. We have recently reported that while the core hub architecture is largely preserved in patients with psychosis, decentralization of the core is noted with strengthening of peripheral hubs, leading to a change in the voxel-wise variance of degree centrality (Palaniyappan and Liddle, 2014). The variance of core degree centrality (VCC) provides a measure of decentralization of the core brain hubs, and has been used to quantify the connectional heterogeneity in social networks (Barocas et al., 2011; Stephenson and Zelen, 1989).

In the present study, we quantified 'loosening of associations' in speech processes using an unbiased speech-graph method in a sample of 56 clinically stable subjects with a psychotic disorder. We studied the differences in speech graph connectedness between schizophrenia and bipolar disorder. Then we verified whether speech connectedness was correlated with two measures of brain structure/function: VCC and gyrification. We also studied correlations of speech connectedness to the psychometric evaluation of formal thought disorder, to processing speed, working memory, and to social and occupational function in patients. This was performed within each diagnostic group as well as trans- diagnostically. Given the multiple association of FTD with biological, behavioural and functional outcomes in psychosis, our hypothesis was that the precise and objective measure of FTD provided by non-semantic graph features would be related to the psychometric evaluation of this class of symptom, to cognitive and global functioning, and - most critically - to brain structure and function.

\section{Methods}

\subsection{Participants}

The sample consisted of 34 patients satisfying DSM-IV criteria for schizophrenia, and 22 patients with DSM-IV bipolar disorder with psychotic features. Patients were recruited from the community based mental health teams (including Early Intervention in Psychosis teams) in Nottinghamshire and Leicestershire, UK. The diagnosis was made in a clinical consensus meeting in accordance with the procedure of Leckman et al.(Leckman et al., 1982), using all available information including a review of case files and a standardized clinical interview (Symptoms and Signs in Psychotic Illness (SSPI) (Liddle et al., 2002b)). All patients were in a stable phase of illness (defined as a change of no $>10$ points in their Global Assessment of Function (GAF, DSM-IV) score, assessed six weeks prior and immediately prior to study participation). No patient had a change in antipsychotic, antidepressant or mood stabilizing medications in the six weeks prior to the study. Subjects with age $<18$ or $>50$, subjects with neurological disorders, current substance dependence, or IQ $<70$ using Quick Test (Ammons and Ammons, 1962) were excluded. The median Defined Daily Dose (WHO, 2003) was calculated separately for antipsychotics (Supplementary Table 1). The study was given ethical approval by the National Research Ethics Committee, Derbyshire, UK. All volunteers gave written informed consent.

\subsection{Clinical assessment}

Patients were interviewed on the same day as the scan and symptom scores assigned according to the SSPI. Two subscales scores (Disorganized thought/language and Impoverished thought/language) were assessed using speech samples in line with the validated procedure for administering Thought Language Index. To generate free speech samples, 3 pictures from Thematic Apperception Test (Murray, 1943) were used in accordance with Sommer et al. (Sommer et al., 2010). Speech samples were audio recorded by two research psychiatrists (LP and VB) and transcribed by researchers (JP, SO) blind to the diagnostic status and symptom burden of the subjects. These four researchers (LP, $\mathrm{VB}, \mathrm{SO}, \mathrm{JP}$ ) participated in several meetings directed by the original author of the scale to receive training in the assessment. Very good inter-rater reliability was demonstrated for 15 speech samples (intraclass correlation for total TLI score $0.83,95 \% \mathrm{CI}=0.59$ to 0.96 ); the remaining speech transcripts were rated by a single rater (SO) blind to diagnosis and symptom burden and neuroimaging findings. We also quantified current occupational and social dysfunction using the Social and Occupational Functioning Assessment Scale (SOFAS) (Goldman et al., 1992) and assessed speed of cognitive processing, a consistent and prominent cognitive deficit in schizophrenia, using the Digit Symbol Substitution Test (Dickinson et al., 2007). DSST was administered using a written and an oral format with a mean DSST score computed from the two formats (Palaniyappan et al., 2013a). N-back test with English alphabets was used to measure working memory (Kirchner, 1958), with performance measured using overall accuracy of responses averaged across 2 sessions (7 task-blocks with randomly presented 0-, 1- and 2-back conditions, each of $30 \mathrm{~s}$ duration with $10 \mathrm{~s}$ interval in-between ( $110 \mathrm{~s} /$ block; 7 blocks/session, 2 sessions in total). Each condition included 4 targets and 11 non-target stimuli with a $2 \mathrm{~s}$ inter-stimulus interval. \{For more details, see (Palaniyappan et al., 2013a)]. 


\subsection{Image acquisition}

Functional MRI datasets were acquired on a 3 Tesla Philips Achieva MRI scanner (Philips, Netherlands) during $10 \mathrm{~min}$ of rest, with eyes open. To enhance sensitivity, dual-echo gradient-echo echo-planar images (GE-EPI) were acquired (Posse et al., 1999), using an eightchannel SENSE head coil with SENSE factor 2 in anterior-posterior direction, TE1/TE2 25/53 ms, flip angle $85^{\circ}, 255 \times 255 \mathrm{~mm}$ field of view, with an in-plane resolution of $3 \mathrm{~mm} \times 3 \mathrm{~mm}$ and a slice thickness of $4 \mathrm{~mm}$, and TR of $2500 \mathrm{~ms}$ ( 40 contiguous axial slices in descending order, 240 time points in total). A magnetisation prepared rapid acquisition gradient echo image (MPRAGE) with $1 \mathrm{~mm}$ isotropic resolution, $256 \times 256 \times 160$ matrix, TR/TE $8.1 / 3.7 \mathrm{~ms}$, shot interval $3 \mathrm{~s}$, flip angle $8^{\circ}$. SENSE factor 2 was also acquired for structural analysis. Out of 56 subjects who underwent clinical assessment, 2 with schizophrenia and 4 with bipolar disorder were excluded due to movement artefacts in either fMRI or structural scans, providing a final sample of 50 subjects for fMRI/structural analysis. There were no differences in the SSPI (total SSPI score mean (SD) in the included group $=10.94(7.96)$. excluded group $=8.33(2.19), p=0.4572$ ) or TLI (total TLI score mean $(\mathrm{SD})$ in the included group $=0.73(0.93)$, excluded group $=0.96(1.29)$, $p=0.7821$ ) between patients who were included or excluded in the analysis.

\section{4. fMRI analysis}

The data was preprocessed using SPM8 (http://www.fil.ion.ucl.ac. $\mathrm{uk} / \mathrm{spm}$ ) and Data Processing Assistant for resting-state fMRI (Chao-Gan and Yu-Feng, 2010). After an initial correction for slice-timing differences, spatial realignment to the first image was carried out. We took several precautions to reduce movement induced confounds. These are detailed in Supplementary Material. A single weighted summation of the dual-echo dynamic time course was obtained for each subject (Posse et al., 1999), followed by retrospective physiological correction using RETROICOR (Glover et al., 2000). Unified segmentation based spatial normalization and smoothing using a Gaussian kernel of $8 \mathrm{~mm}$ FullWidth at Half Maximum was carried out. Following this, linear detrending and filtering using a band pass filter $(0.01-0.08 \mathrm{~Hz})$ was done to eliminate low frequency fluctuations and high frequency noise. Finally, variance accounted for by six head motion parameters, global mean signal, white-matter signal and CSF signal was removed by regression before conducting the degree centrality (DC) analysis.

Pre-processed data was analysed by deriving degree of centrality measure for every grey matter voxel using the cortical hub analysis procedure described by Buckner et al. (Buckner et al., 2009), and implemented in the REST software (Chao-Gan and Yu-Feng, 2010). For each voxel, we extracted the BOLD time course and correlated with every other voxel in the brain. For each voxel $j$ the number of strong voxel-to-voxel correlations (defined as correlation coefficient $r>0.25$ ) was computed to determine the DC of $\mathrm{j}$. The threshold of 0.25 was chosen to avoid the inclusion of voxels that had low functional connectivity with the index voxel. For each subject, a map with DC values for every grey matter voxel was obtained. These maps were then ztransformed to enable group comparisons. Core hubs were identified using the entire sample as previously reported (Palaniyappan and Liddle, 2014) (Supplementary Tables 2 and 3; Suppl. Fig. 1). For voxels contained within the core hubs the voxelwise variance of normalized degree centrality of the core (VCC) was computed for each subject.

\subsection{Surface extraction}

Cortical surfaces were reconstructed from MPRAGE images using FreeSurfer version 5.1.0. The pre-processing was performed using standard procedures as described by Dale et al. (Dale et al., 1999). Following skull-stripping and intensity correction, the grey-white matter boundary for each cortical hemisphere was determined using tissue intensity and neighbourhood constraints. Total surface area (TSA = sum of right and left hemispheric area) was computed from reconstructed grey-white boundary. Vertexwise cortical folding pattern was quantified using local gyrification index, using the method advocated by Schaer et al. (Schaer et al., 2008) and described in detail in our previous study using an overlapping sample (Palaniyappan and Liddle, 2014). A global index of gyrification was obtained by computing the mean of the local gyrification index (LGI) values from each vertex in each subject. A mean gyrification index (MGI) $>1$ indicates a larger folded area ('buried' surface) compared to the outer circumference of the cortex ('visible' surface').

\subsection{Speech graph analysis}

Graphs were constructed from each subject's speech samples obtained during 3 instances of $1 \mathrm{~min}$ freely generated speech on the presentation of the pictures for the TLI. The speech samples were blindly transcribed by 2 researchers (JP and SO). Next, the samples were converted to graphs by a rater who was blind to diagnostic status (NBM), using an automated algorithm freely available online (SpeechGraphs, http://www.neuro.ufrn.br/softwares/speechgraphs) (Mota et al., 2014). In these graphs each word corresponded to a node, and each temporal link between words corresponded to an edge (Fig. 1A). As a result, each word trajectory corresponded to an unweighted directed graph. To quantify graph connectedness, we used the SpeechGraphs software to calculate 2 connectedness speech graph attributes (Fig. 1A), which comprised connected components (LCC and LSC) (Fig. 1A). The LCC (largest connected component) is the total of nodes within the largest openly connected component of the graph, in which nodes are linked by at least one directed path. The LSC (largest strongly connected component) is the total of nodes within the largest closed component of the graph, in which any pair of nodes is linked by a direct or indirect path, and therefore mutually reachable, i.e., node "a" reaches node " $b$ " and node " $b$ " reaches node " $a$ ".

In order to control for verbosity differences, connectedness attributes were calculated in sliding windows of 30 words (with 15-word overlap among consecutive windows) (Fig. 1B) and averaged over all windows from each report. To verify how close to randomness was the connectedness (LCC and LSC) of each graph from each word window, we performed 100 shuffles in the sequence of each set of words (Fig. 1C). Then we averaged the connectedness attributes of all random graphs and all word windows, producing a mean random value of LCC and LSC for each text. The original and the random values of LCC and LSC were averaged over the 3 images reports, generating one original mean value of LSC and LCC and one random value of LSC and LCC per subject. Then we calculated the ratio of original connectedness (LCC or LSC) by random connectedness (random LCC or random LSC) to verify how close to randomness this original report was (ratio closer to 1 means closer to randomness). We called these measures LCCr and LSCr. A subject, whose speech output, irrespective of number of words spoken, appears to have random links with low degree of goal directedness, will have LCCr value close to 1 . On the other hand a subject whose speech makes no referential ties, and is simply a collection of random words temporally linked together, will have an $\mathrm{LSCr}$ value close to 1 .

Previous studies showed that patients with schizophrenia diagnosis produced reports with lower speech connectedness (smaller LCC and LSC) reaching levels statistically similar to those of random speech connectedness (LSCr), when reporting a dream or an affective image (Mota et al., 2017), but not when reporting a spontaneous recollection of waking activities (Mota et al., 2014). Since the contents of the verbal reports in the present study were different from those mentioned above, and given the difference in the languages used (previous studies investigated native Portuguese speakers, the present study assessed native English speakers), we calculated the four measures of speech connectedness to verify whether the results could be replicated. 
A

It is a sensible lady with two books. Behind her is
a lady and a man with a horse. And some she would!
And a mountain and clear sky.

No

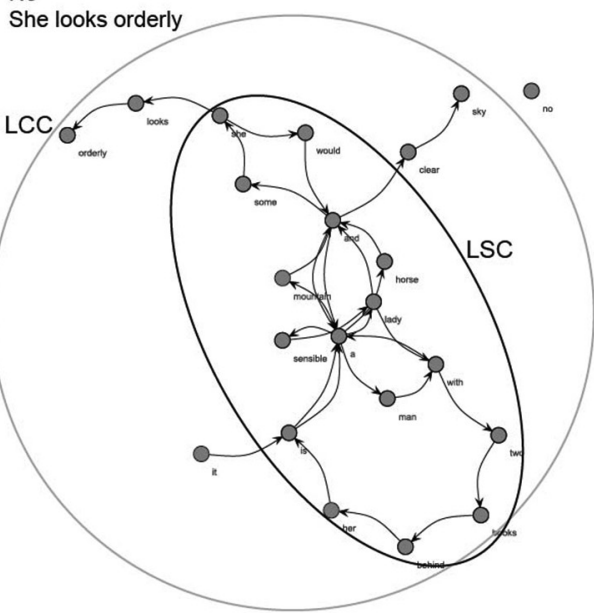

C
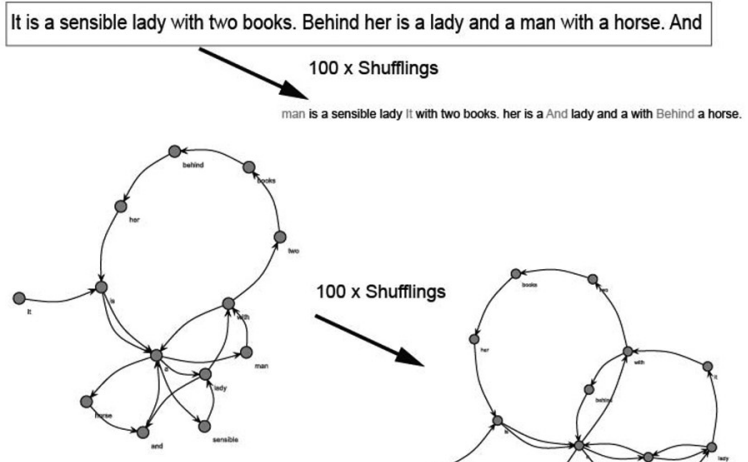

Original Graph (LCC and LSC)

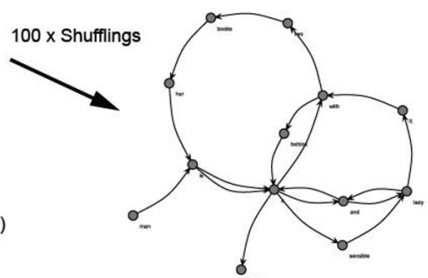

Mean LCCr and LSCr of 100 Random Graphs

B

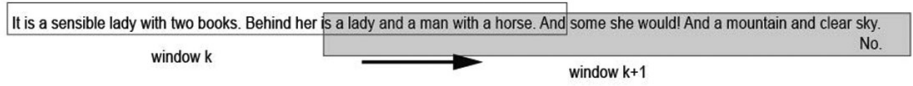

Mean value $=\frac{\text { Graph attribute value per window }}{\text { Number }}$

Number of windows

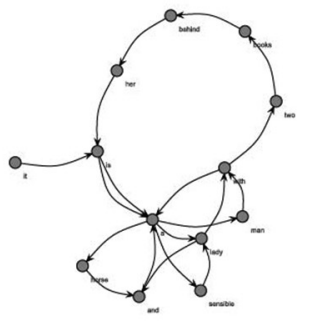

graph for window $k$

D

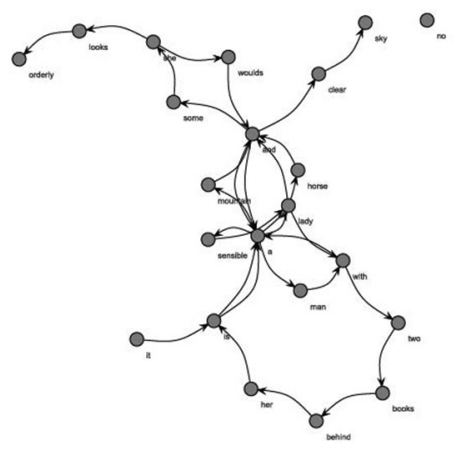

Schizophrenia Group Example

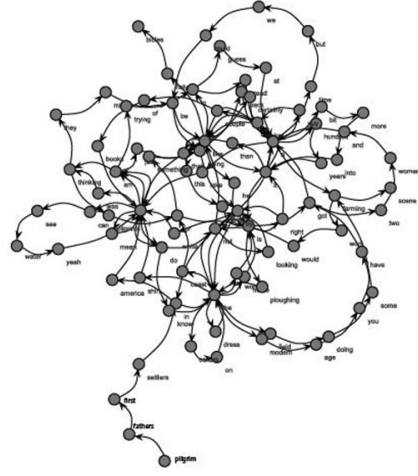

Bipolar Group Example

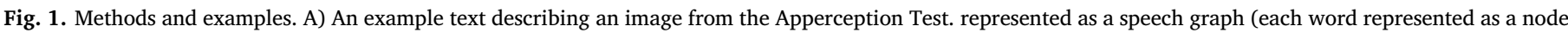

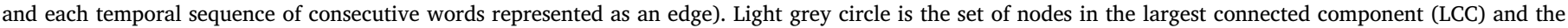

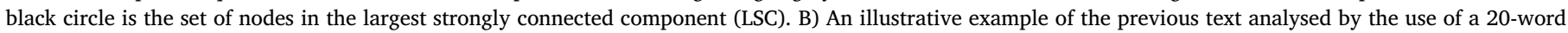

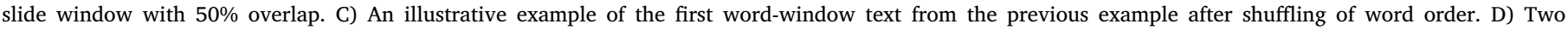
representative examples of the Schizophrenia and Bipolar groups.

\subsection{Statistical analysis}

The graph attributes (originals LCC and LSC, and ratios LCCr and LSCr) were compared between the two groups using a Rank-sum test.

\subsection{Canonical correlations}

In order to correlate different sets of speech graph connectedness measures to other brain measures, psychometric evaluation of thought disorder, global functioning, and aspects of cognitive performance, we calculated canonical correlations (Das and Sen, 2014). To avoid collinearity, we performed multicollinearity diagnosis and used only sets of measures with conditioning index lower than 30. After that, we considered LCC, LSC and LSCr as the set for speech connectedness, VCC and LGI for brain structure/function, two sub-scales indexes of TLI (Disorganization and Impoverishment) for psychometric evaluation of thought disorder, GAF and SOFAS for global functioning, DSST, and nback (0, 1 and 2-back) respectively for processing speed and working memory, two different aspects of cognitive performance. The main hypothesis was that speech connectedness should be correlated with psychometric, behavioural, functional and biological correlates of FTD; this was assessed with the canonical correlation. This analysis allowed us to verify whether there was a correlation between the sets of measures collected and how much variance we could explain from these relationships. The canonical coefficients define the relationship between the initial variable and the canonical variable (showed on Fig. 2B and $\mathrm{C}, \mathrm{X}$ and $\mathrm{Y}$ axis). Also, we studied Spearman correlations for each pair of variables to better understand the direction of each relationship. As each of the 5 sets of assessments (speech connectedness, brain structure/function, psychometric evaluation of thought disorder, aspects of cognitive performance and global functioning) were correlated with the other 4 sets, and as each set was not expected to be independent from each other (given their associations), the canonical correlation results were considered significant after Bonferroni correction for 4 comparisons ( $p<0.0125$ ). This threshold aimed to minimize false positives without increasing false negatives. The canonical correlations were also performed within each diagnostic category (Schizophrenia and Bipolar Disorder). For isolated Spearman correlations we considered $p<0.0062$, with Bonferroni correction for 8 comparisons (considering VCC, LGI, Disorganization, Impoverishment, GAF, SOFAS, DSST, n-back across two sessions). 
A
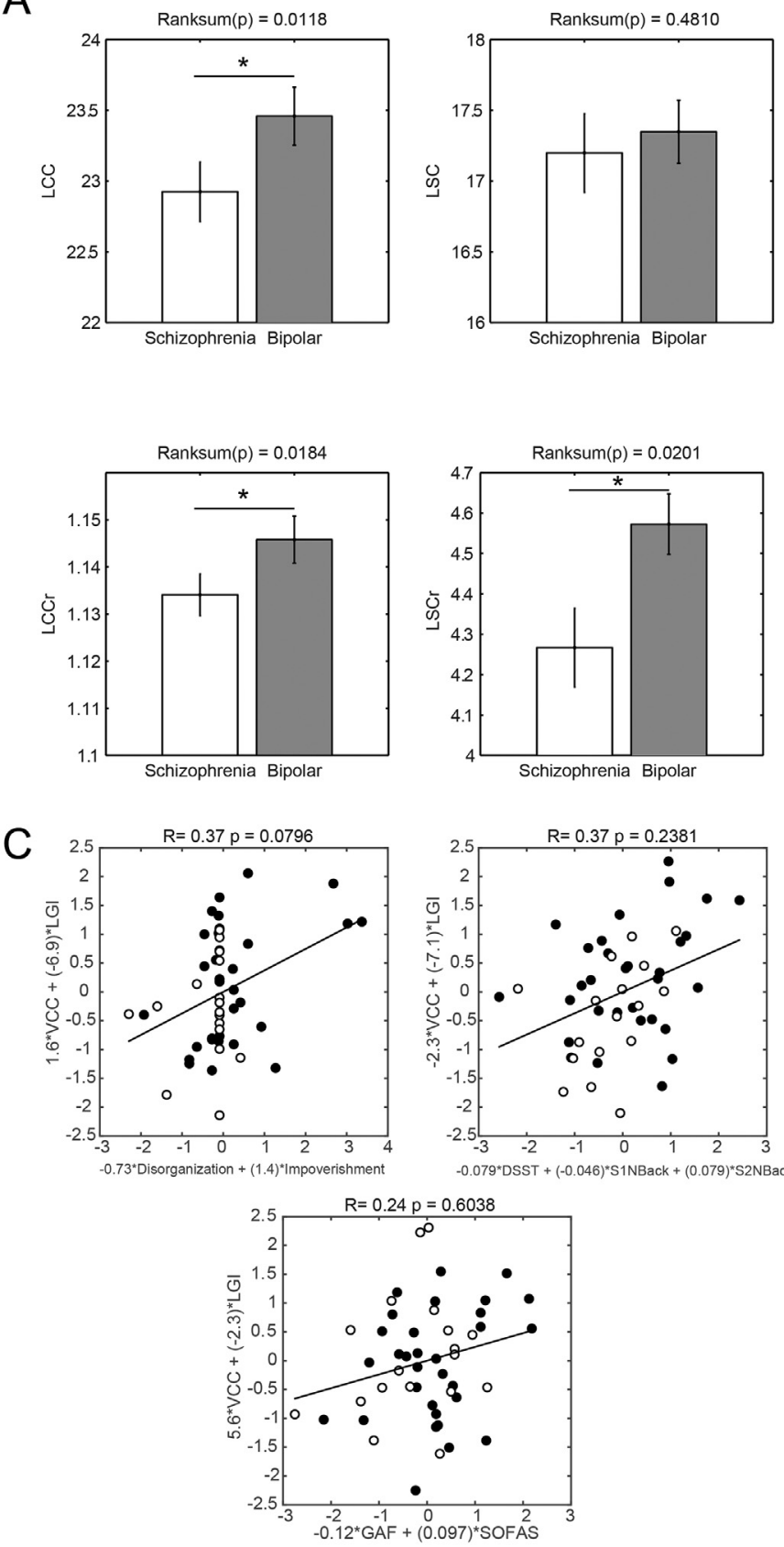

$\mathrm{B}$
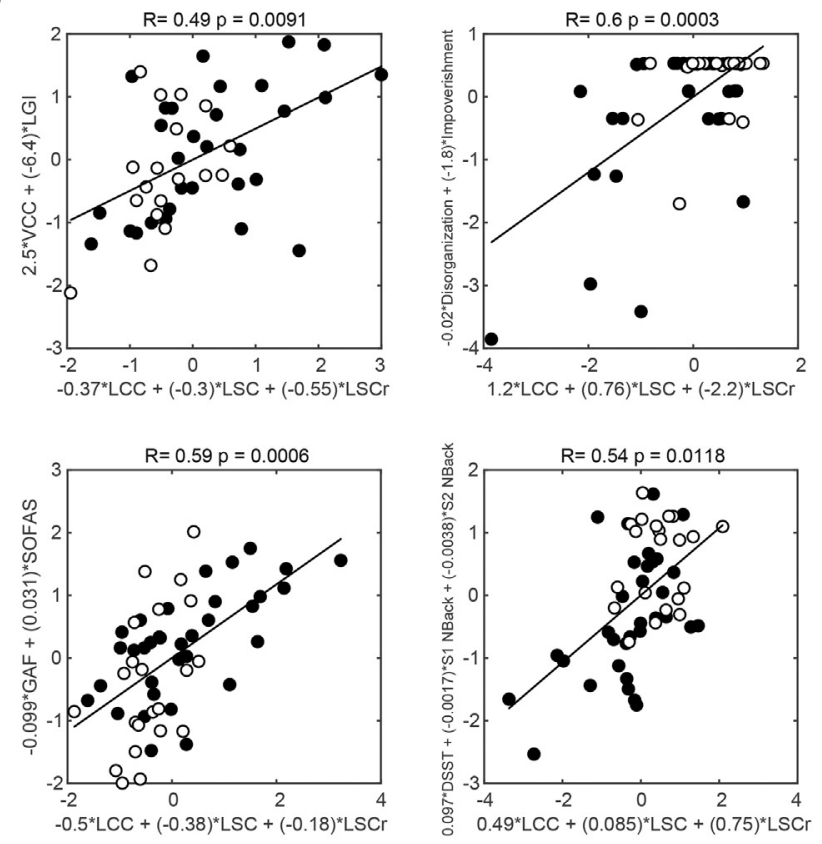

$\mathrm{D}$

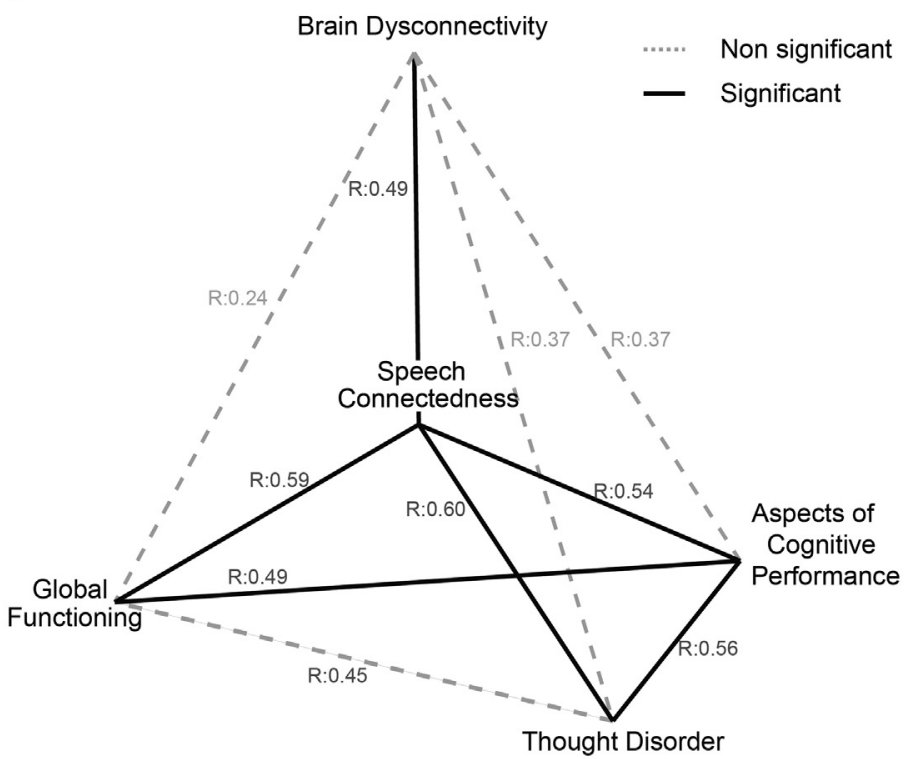

Fig. 2. Speech connectedness (lower in Schizophrenia) is the only behavioural measure to correlate with brain structure/function. A) Schizophrenia group presents lower connectedness (LCC, LCCr and LSCr, details in methods) than Bipolar group. B) Speech connectedness is correlated with brain structure/function (measured by VCC - variance of the degree centrality of the core hubs and LGI - gyrification index), with sub-scales of TLI (Disorganization and Impoverishment), with global functioning (measured by GAF and SOFAS) and aspects of cognitive performance (measured by DSST and n-Back). Canonical variates of each set of variables indicated on $\mathrm{X}$ and $\mathrm{Y}$ axis, followed by respective canonical coefficients (illustrative of the relationship between each initial variable and the canonical variable). Subjects from Bipolar group in dark dots, and from Schizophrenia group in white dots. C) None of the other behavioural measures (such as TLI, or global functioning or aspects of cognitive performance) were correlated with brain structure/function. Canonical variates of each set of variables indicated on X and Y axis, followed by respective coefficients. Subjects from Bipolar group in dark dots, and from Schizophrenia group in white dots. D) Schematic summary of main results illustrating the main correlations searched in this work.

\section{Results}

The demographic and clinical features of the two groups are shown in Table 1. There was a statistical difference related to socioeconomic status between the groups, but no other measure related to age, sex, IQ, equivalent dose of medication, handedness or disease duration was found to be statistically different. There were no significant differences related to psychometric evaluation, global functioning, and aspects of cognitive performance or brain structure/function between the Schizophrenia and Bipolar groups.

\subsection{Speech graph analysis}

From mathematical definitions of the four speech connectedness measures (LCC, LSC, LCCr and LSCr) we understand that they measure similar aspects of what we call here "speech connectedness". To observe 
Table 1

Demographic and clinical features: IQ: intelligence quotient; CPZeq dose: equivalent dose of antipsychotic medication compared to chlorpromazine; NSSEC: National Statistics - Socio Economic Status; SD: standard deviation; SSPI: Symptoms and Signs of Psychotic Illness; TLI: Thought Language Index; VCC: Variance of the degree centrality of the core hubs; SOFAS: Social and Occupational Functioning Assessment Scale; GAF: Global Assessment of Function; DSST: Digit Symbol Substitution Score; N-Back: boldface indicates $p<0.05$.

\begin{tabular}{|c|c|c|c|}
\hline Data & $\begin{array}{l}\text { Patients with } \\
\text { bipolar disorder } \\
(n=22)\end{array}$ & $\begin{array}{l}\text { Patients with } \\
\text { schizophrenia } \\
(n=34)\end{array}$ & $\begin{array}{l}P \text { values }\left(X^{2}\right. \\
\text { or Ranksum) }\end{array}$ \\
\hline Gender (male/female) & $14 / 8$ & $29 / 5$ & 0.0608 \\
\hline $\begin{array}{l}\text { Handedness (right/ } \\
\text { left) }\end{array}$ & $20 / 2$ & $29 / 5$ & 0.5349 \\
\hline Mean age in years (SD) & $34.6(10.4)$ & $32.9(8.9)$ & 0.7945 \\
\hline Mean IQ (SD) & $102.86(13.88)$ & $95.12(13.18)$ & 0.7075 \\
\hline $\begin{array}{l}\text { Mean disease duration } \\
\quad \text { (SD) }\end{array}$ & $11.05(8.13)$ & $8.82(6.72)$ & 0.7426 \\
\hline Mean CPZeq dose (SD) & 209.5(228.18) & $582.81(505.56)$ & 0.2189 \\
\hline $\begin{array}{l}\text { Mean parental NS-SEC } \\
\quad \text { (SD) }\end{array}$ & $1.67(1.13)$ & $2.52(1.52)$ & 0.0436 \\
\hline $\begin{array}{l}\text { Mean total SSPI score } \\
\text { (SD) }\end{array}$ & $8.05(7.99)$ & $12.26(7.18)$ & 0.4192 \\
\hline $\begin{array}{l}\text { Mean total TLI score } \\
\text { (SD) }\end{array}$ & $0.66(1.14)$ & $0.81(0.8)$ & 0.5283 \\
\hline $\begin{array}{l}\text { Mean TLI } \\
\text { disorganization } \\
\text { (SD) }\end{array}$ & $0.53(0.96)$ & $0.40(0.55)$ & 0.8754 \\
\hline $\begin{array}{l}\text { Mean TLI } \\
\text { impoverishment } \\
\text { (SD) }\end{array}$ & $0.13(0.30)$ & $0.41(0.66)$ & 0.5027 \\
\hline Mean LGI (SD) & $2.99(0.12)$ & $2.95(0.16)$ & 0.4968 \\
\hline Mean VCC (SD) & $0.82(0.18)$ & $0.77(0.15)$ & 0.6711 \\
\hline Mean SOFAS (SD) & $60.32(13.36)$ & $54.62(12.82)$ & 0.7431 \\
\hline Mean GAF (SD) & $56.68(14.08)$ & $48.79(10.8)$ & 0.7053 \\
\hline Mean DSST (SD) & $50.48(8.08)$ & $41.97(10.15)$ & 0.2532 \\
\hline Mean n-back (SD) & 87.61(11.87) & $85.48(14.33)$ & 0.4888 \\
\hline
\end{tabular}

empirically on this sample the magnitude of the shared co-variance between those measures, we calculated $\mathrm{R}$ square $\left(\mathrm{R}^{2}\right)$ and $p$ values of each correlation pair. All different combinations showed $\mathrm{p}$ values smaller than 0.0031 , confirming that all measures were correlated, but the level of co-variance spanned between $\mathrm{R}^{2} 0.22$ to $0.99\left(\mathrm{R}^{2}\right.$ : LCC $\mathrm{x}$ LSC $=0.99 ; \quad$ LCC $\quad x \quad$ LCCr $=0.22, \quad$ LCC $\quad x \quad$ LSCr $=0.38 ; \quad$ LSC $\quad x$ $\mathrm{LCCr}=0.23$; LSC $\times$ LSCr $=0.39$; LCCr $\times$ LSCr $=0.81$ ), which means that those measures are not exactly the same. We interpreted our results after taking this point into account, with emphasis on measures corrected for randomness.

Speech connectedness indexed by LCC, LCCr and LSCr was significantly different between the 2 groups. In general, patients with schizophrenia produced less connected reports, with speech connectedness closer to chance when compared to bipolar disorder (lower LCC, LCCr and LSCr, far from 1) (Fig. 1D and 2A), indicating that in schizophrenia patients the referential ties as well as the goal directedness were reduced when compared to bipolar disorder.

\subsection{Correlations with brain structure/function}

We found significant canonical correlation between speech connectedness and brain structure/function, which explained nearly $25 \%$ of the variance ( $R=0.49, p=0.0091$; Fig. $2 \mathrm{~B}$, D and Table 2). Using the Spearman correlation, VCC (during resting state) was anti-correlated with LCC and LCCr (Table 3), but there was no significant correlation with LGI, only a trend between LGI and LSCr (Table 3). No other significant canonical correlations between brain structure/function (LGI and VCC) and psychometric evaluations (formal thought disorder measured by TLI), global functioning assessments (GAF and SOFAS) or aspects of cognitive performance (DSST, N-back) were
Table 2

Canonical correlations: Brain Markers (VCC: Variance of the degree centrality of the core hubs; LGI: Gyrification index); Speech Connectedness (LCC: Largest connected component; LSC: Largest strongly connected component; LSCr: ratio LSC/mean LSC in 100 random graphs); Global Functioning (GAF: Global Assessment of Function; SOFAS: Social and Occupational Functioning Assessment Scale); Thought Disorder (DIS: Disorganized thought/language; IMP: Impoverished thought/language); Cognitive Performance (DSST: Digit Symbol Substitution Score; n-back: working memory test); boldface indicates $p<0.0125$.

\begin{tabular}{lll}
\hline Canonical correlation & $\mathrm{R}$ & $\mathrm{P}$ \\
\hline Speech connectedness $\times$ Brain markers & 0.49 & $\mathbf{0 . 0 0 9 1}$ \\
Speech connectedness $\times$ Global functioning & 0.59 & $\mathbf{0 . 0 0 0 6}$ \\
Speech connectedness $\times$ Thought disorder & 0.60 & $\mathbf{0 . 0 0 0 3}$ \\
Speech connectedness $\times$ Aspects of cognitive performance & 0.54 & $\mathbf{0 . 0 0 4 7}$ \\
Brain markers $\times$ Thought disorder & 0.37 & 0.0796 \\
Brain markers $\times$ Aspects of cognitive performance & 0.32 & 0.1860 \\
Brain markers $\times$ Global functioning & 0.24 & 0.6038 \\
Global functioning $\times$ Thought disorder & 0.45 & 0.0195 \\
Global functioning $\times$ Aspects of cognitive performance & 0.49 & $\mathbf{0 . 0 0 7 3}$ \\
Thought disorder $\times$ Aspects of cognitive performance & 0.45 & $\mathbf{0 . 0 1 2 0}$ \\
\hline
\end{tabular}

observed (Fig. 2C, D and Table 2).

\subsection{Correlations with clinical assessment}

There was a significant canonical correlation between speech connectedness and the psychometric evaluation of thought disorder, which explained $36 \%$ of the variance ( $R=0.6, p=0.0003$; Fig. $2 \mathrm{~B}$, D and Table 2). Impoverishment and LSCr showed negative Spearman correlation (Table 3): More severe symptoms were associated with largest closed loops closer to random. A significant canonical correlation occurred between speech connectedness and global functioning, which explained $34 \%$ of the variance $(R=0.59, p=0.0006$; Fig. $2 \mathrm{~B}$, D and Table 2), with positive Spearman correlations between GAF and LCC, LSC and LSCr (Table 3): Higher scores on GAF were associated with more nodes in the largest closed and open components, and closed components farther from what would be expected by chance. Importantly, speech connectedness showed significant canonical correlation with aspects of cognitive performance, explaining $29 \%$ of the variance ( $R=0.54, p=0.0047$; Fig. $2 \mathrm{~B}, \mathrm{D}$ and Table 2$)$, with positive Spearman correlations between DSST and LCC, LCCr and LSCr (Table 3). This means that better performance was correlated with larger deviations of open and closed components in relation to randomness. There were significant canonical correlations among all behavioural assessments, except for psychometric evaluation and global functioning (Table 2).

\subsection{Correlations within the schizophrenia and bipolar groups}

To investigate potential group specificities we separately calculated the same canonical correlations for each diagnostic group (schizophrenia and bipolar disorder) (Suppl. Fig. 2). Only for the schizophrenia group we found a significant relationship between i) brain function and speech, and ii) social function and speech. The correlations between speech connectedness and thought disorder/cognitive tests were not significant for any of the groups separately (Suppl. Fig. 2). It is important to consider that the $R$ values of the canonical correlations obtained were very similar to those obtained when considering all subjects together, and therefore the reduction in $\mathrm{N}$ most likely explains the increase in $p$ values.

\section{Discussion}

The results suggest that the application of graph theory to analyse the connectedness of speech samples in clinically stable patients can 
Table 3

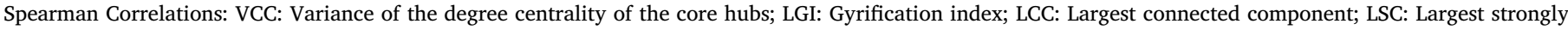

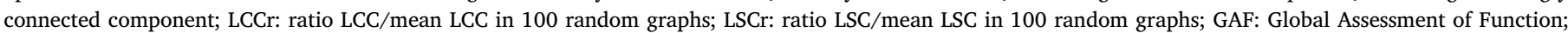

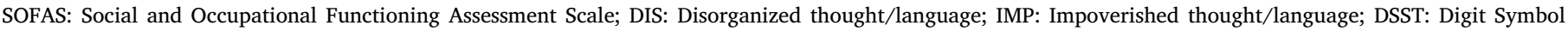
Substitution Score; N-Back: working memory test, boldface indicates $p<0.0062$.

\begin{tabular}{|c|c|c|c|c|c|c|c|c|c|c|c|c|c|c|c|c|}
\hline \multirow{2}{*}{$\begin{array}{l}\text { Spearman } \\
\text { Connectedness }\end{array}$} & \multicolumn{2}{|l|}{ VCC } & \multicolumn{2}{|l|}{ LGI } & \multicolumn{2}{|l|}{ DIS } & \multicolumn{2}{|l|}{ IMP } & \multicolumn{2}{|l|}{ GAF } & \multicolumn{2}{|c|}{ SOFAS } & \multicolumn{2}{|l|}{ DSST } & \multicolumn{2}{|c|}{ N-Back } \\
\hline & Rho & $\mathrm{p}$ & Rho & $\mathrm{p}$ & Rho & $\mathrm{p}$ & Rho & $\mathrm{p}$ & Rho & $\mathrm{p}$ & Rho & $\mathrm{p}$ & Rho & $\mathrm{p}$ & Rho & $\mathrm{p}$ \\
\hline LCC & -0.40 & 0.0035 & 0.25 & 0.0662 & -0.12 & 0.3790 & -0.27 & 0.0447 & 0.41 & 0.0018 & 0.14 & 0.3118 & 0.39 & 0.0027 & 0.14 & 0.2978 \\
\hline LSC & -0.04 & 0.7812 & 0.21 & 0.1227 & -0.08 & 0.5448 & -0.33 & 0.0131 & 0.48 & 0.0002 & 0.28 & 0.0343 & 0.29 & 0.0312 & 0.03 & 0.8186 \\
\hline LCCr & -0.42 & 0.0025 & 0.14 & 0.2945 & -0.21 & 0.1154 & -0.21 & 0.1277 & 0.32 & 0.0148 & 0.06 & 0.6689 & 0.36 & 0.0063 & 0.14 & 0.3166 \\
\hline LSCr & -0.24 & 0.0949 & 0.32 & 0.0162 & -0.14 & 0.3132 & -0.38 & 0.0041 & 0.56 & 0.0000 & 0.32 & 0.0175 & 0.46 & 0.0003 & 0.07 & 0.6293 \\
\hline
\end{tabular}

help to establish the relationship of this pathological phenomenology with biological markers. Speech connectedness (the amount of nodes or words engaged in the largest open and closed components of word trajectory graphs, as well as the deviation from random word graphs made with the same set of words) is correlated to measures of cortical development and dysconnectivity. Speech connectedness explained a core cognitive deficit and functional outcome in patients, irrespective of diagnostic boundaries.

In the present study, we note that the less connected the speech, the lower the scores in global functioning (stronger results with GAF) and processing speed (digit-symbol test). The relationship between social outcome and impoverished speech has been demonstrated previously both in chronic schizophrenia (Bowie and Harvey, 2008) and in adolescents at high risk for schizophrenia (Bearden et al., 2011). This reaffirms the consistent associations shown between communication disturbances and predictors of social functioning such as social cognition and engagement (Bowie et al., 2011). In their meta-analysis of neuro-cognition in relation to clinical syndromes, Dominguez et al. (Dominguez Mde et al., 2009) noted a significant association between disorganization and processing speed deficits. Our observations suggest that this relationship is mediated by the presence of impoverished thought. While we observed a robust relationship between speech connectedness and GAF, the correlation with SOFAS was somewhat weaker, not reaching our stringent thresholds for statistical significance. As GAF factors in current psychopathology as well as social function, the observed relationship may not be specific to social functioning.

In previous work with Portuguese-speaking patients it was found that speech connectedness measured by graph analysis was strongly anti-correlated with severity in negative and cognitive symptoms measured by psychometric evaluation in chronic patients (Mota et al., 2014) and even before the formal diagnosis in recent onset psychosis patients (Mota et al., 2017), helping differential diagnosis of Schizophrenia in both situations (Mota et al., 2017; Mota et al., 2014; Mota et al., 2012). We replicate these results, also finding a more connected speech structure in patients with Bipolar Disorder diagnosis in comparison to the Schizophrenia diagnosis, even in native speakers of another language (English). This corroborates the language invariance of this method (Mota et al., 2014). These findings are in line with previous works suggesting that in schizophrenia, less syntactically complex utterances are prevalent, with a lack of coherent discourse structure (Hoffman et al., 1986; Thomas et al., 1996; Fraser et al., 1986; King et al., 1990). This supports the notion that the thought disorder has a dimensional distribution across psychotic disorder, with selected components of this dimension being more pronounced in schizophrenia than in bipolar disorder (Perlini et al., 2012).

Extending previous work (Mota et al., 2017; Mota et al., 2014), we have also identified the relationship between abnormalities in the language structure and the clinician's perception of thought disorder. A less connected speech output, as measured by low values of connectedness speech graphs attributes, maps on to an impoverished thought/language score as rated by clinicians. Taken together, these evidences indicate that connectedness of speech, particularly in comparison to randomness, is associated to poverty in thought process expressed through language.

Besides this relationship between graph analysis of speech and psychometric evaluation, we notice that speech connectedness explains $24 \%$ of the variance in brain structure/function, but not between the latter and the psychometric evaluation of thought disorder (TLI). The naturalistic, automated method of quantifying speech connectedness indexes the variation necessary to relate thought disorder and brain structure/function. Specifically, reduced goal directedness (i.e. connectedness similar to random set of words, low LCCr) of the speech structure was associated with higher variance in the centrality. This suggests that with a reduction in the integrity of the core functional hubs (especially left inferior parietal, superior temporal, anterior and posterior cingulate and insula, see Supplement Table S2), reflected by an increase in voxel-wise variance of the centrality, speech structure becomes disorganized, approaching randomness. To our knowledge, this is the first time that a relationship between a measure reflecting the decentralization of brain's functional network architecture and thought disorder has been reported. This highlights the potential usefulness of the combined clinical and automated graphical assessment of speech disturbances in psychosis. Furthermore, the observation that several speech/language features predict functional outcome and measures of brain structure/function after allowing for variance in diagnosis supports the hypothesis that some pathological features are distributed in a continuum across psychotic disorders.

The trend of positive correlation between speech graph connectedness and gyrification indicates that subjects with a neurodevelopmental disturbance indexed by reduced gyrification produce speech with lower degree of referential ties approaching randomness (low LSCr). We have previously reported that the most significant reduction in gyrification affects the fronto-insular cortex and the superior temporal cortex in psychosis (Palaniyappan and Liddle, 2014; Palaniyappan and Liddle, 2012; Palaniyappan et al., 2013b), in line with several previous studies suggesting a relationship between the dysfunction of these brain regions and formal thought disorder. In the context of the current findings, the relationship between specific components of thought disorder and the gyrification patterns of these regions require further study. We have previously shown a relationship between speech connectedness and the typical development of intelligence quotient and theory of mind abilities, which allows a prediction of reading acquisition in typical developing children (Mota et al., 2016b) - but note that this study did not investigate gyrification. Also a slow asymptotic development of speech connectedness, reaching asymptotic stable values around 13 years of schooling, was observed in typical developing individuals, but not in a psychotic population, despite schooling (Mota et al., 2016a).

While the correlations with speech connectedness across diagnostic groups were significant, most of the correlations within each diagnostic group were not. No correlations at all were significant within the bipolar group, and only correlations with brain function and global functioning were significant within the schizophrenia group. This could 
be interpreted to support the assumption that the relationships with speech connectedness are restricted to the latter group. But since the $\mathrm{R}$ values found on Bipolar group analysis were similar to the $R$ values found when considering the entire sample, and given that sample reduction impacts $p$ values, it is clear that additional studies with larger samples are necessary to further investigate this point.

A major limitation of the current study is that we lacked equivalent speech sample data for healthy controls. As a result, we are unable to compare the speech graph properties with apparently normal individuals. Previous studies have shown that the presence of disordered thought form is not unique to psychosis and can occur in healthy individuals as well as people with other psychiatric disorders (Sommer et al., 2010; Bearden et al., 2011). Most of our patients were medicated; this could have reduced the severity and the variance of thought disturbances. Yet, as shown in our previous study using this sample (Palaniyappan and Liddle, 2014), we did not find any linear relationship between prescribed medications (Supplement Table 1) and the degree of abnormalities in gyrification or centrality. Nevertheless, we cannot completely rule out the confounding effects of medications. Another limitation is the use of GAF, which is confounded by the mixture within its rating system of psychopathological symptoms and functional measures.

Neuroimaging of symptoms in psychosis is widely believed to be a powerful means to further understand psychiatric disorders at a systems level (Redpath et al., 2013). The speech graph approach employed here provides an automated, unbiased means of quantifying the subtle speech disorders in psychosis with a short duration of assessment. In combination with clinical ratings, this approach not only predicts realworld outcome measures but also reveals the neurobiological underpinnings of thought disorders, thus providing a robust means to study the pathophysiology of psychosis.

\section{Financial support}

This work was funded by the Medical Research Council (UK) Grant Number: G0601442. L Palaniyappan is supported by the Canadian Institute of Health Research (Foundation Grant 375,104), Bucke Family Fund and the Academic Medical Organization of South Western Ontario. SR and MC were funded by Conselho Nacional de Desenvolvimento Científico e Tecnológico (CNPq) (grants 308,775/ 2015-5, 408,145/2016-1 and 310,712/2014-9), STIC AmSud 062/ 2015 and FAPESP Center for Neuromathematics (grant 2013/07699-0, S. Paulo Research Foundation FAPESP). NM, SR and MC supported by Boehringer-Ingelheim (grants 270,906 and 270,561).

\section{Conflicts of interest}

L Palaniyappan received speaker fees from Otsuka and educational grant from Janssen in 2017. P F Liddle has received honoraria for academic presentations from Janssen-Cilag and Bristol Myers Squibb; and has taken part in advisory panels for Bristol Myers Squibb. NBM, SR and MC receive support from Boehringer-Ingelheim since 2017. All other authors declare no conflict of interest.

\section{Ethical standards}

The authors assert that all procedures contributing to this work comply with the ethical standards of the relevant national and institutional committees on human experimentation and with the Helsinki Declaration of 1975, as revised in 2008. The authors assert that all procedures contributing to this work comply with the ethical standards of the relevant national and institutional guides on the care and use of laboratory animals.

\section{Appendix A. Supplementary data}

Supplementary data to this article can be found online at https:// doi.org/10.1016/j.pnpbp.2018.07.007.

\section{References}

Ammons, R.B., Ammons, C.H., 1962. The quick test (QT): provisional manual. Psychol. Rep. 11, 111-161.

Andreasen, N.C., Grove, W.M., 1986. Thought, language, and communication in schizophrenia: diagnosis and prognosis. Schizophr. Bull. 12, 348-359.

Barocas, A., Ilany, A., Koren, L., Kam, M., Geffen, E., 2011. Variance in centrality within rock hyrax social networks predicts adult longevity. PLoS ONE 6, e22375.

Bearden, C.E., Wu, K.N., Caplan, R., Cannon, T.D., 2011. Thought disorder and communication deviance as predictors of outcome in youth at clinical high risk for psychosis. J. Am. Acad. Child Adolesc. Psychiatry 50, 669-680.

Bleuler, E., 1950. Dementia Praecox: Or The Group of Schizophrenias. International Universities Press.

Bowie, C.R., Harvey, P.D., 2008. Communication abnormalities predict functional out comes in chronic schizophrenia: differential associations with social and adaptive functions. Schizophr. Res. 103, 240-247.

Bowie, C.R., Gupta, M., Holshausen, K., 2011. Disconnected and underproductive speech in schizophrenia: unique relationships across multiple indicators of social functioning. Schizophr. Res. 131, 152-156.

Buckner, R.L., Sepulcre, J., Talukdar, T., Krienen, F.M., Liu, H., Hedden, T., AndrewsHanna, J.R., Sperling, R.A., Johnson, K.A., 2009. Cortical hubs revealed by intrinsic functional connectivity: mapping, assessment of stability, and relation to Alzheimer's disease. J. Neurosci. 29, 1860-1873.

Cavelti, M., Homan, P., Vauth, R., 2016. The impact of thought disorder on therapeutic alliance and personal recovery in schizophrenia and schizoaffective disorder: An exploratory study. Psychiatry Res. 239, 92-98.

Cavelti, M., Kircher, T., Nagels, A., Strik, W., Homan, P., 2018. Is formal thought disorder in schizophrenia related to structural and functional aberrations in the language network? A systematic review of neuroimaging findings. Schizophr. Res.

Chao-Gan, Y., Yu-Feng, Z., 2010. DPARSF: A matlab toolbox for "Pipeline" data analysis of resting-state fMRI. Front. Syst. Neurosci. 4, 13.

Chapman, L.J., Chapman, J.P., 1973. Disordered Thought in Schizophrenia. AppletonCentury-Crofts, East Norwalk, CT, US.

Dale, A.M., Fischl, B., Sereno, M.I., 1999. Cortical surface-based analysis. I. Segmentation and surface reconstruction. NeuroImage 9, 179-194.

Das, S., Sen, P.K., 2014. Canonical Correlation. Wiley StatsRef: Statistics Reference Online. John Wiley \& Sons, Ltd.

Demjaha, A., Weinstein, S., Stahl, D., Day, F., Valmaggia, L., Rutigliano, G., De Micheli, A., Fusar-Poli, P., Mcguire, P., 2017. Formal thought disorder in people at ultra-high risk of psychosis. BJPsych Open 3, 165-170.

Dickinson, D., Ramsey, M.E., Gold, J.M., 2007. Overlooking the obvious: a meta-analytic comparison of digit symbol coding tasks and other cognitive measures in schizophrenia. Arch. Gen. Psychiatry 64, 532-542.

Dominguez Mde, G., Viechtbauer, W., Simons, C.J., Van Os, J., Krabbendam, L., 2009. Are psychotic psychopathology and neurocognition orthogonal? A systematic review of their associations. Psychol. Bull. 135, 157-171.

Dubois, J., Benders, M., Borradori-Tolsa, C., Cachia, A., Lazeyras, F., Ha-Vinh Leuchter, R., Sizonenko, S.V., Warfield, S.K., Mangin, J.F., Huppi, P.S., 2008. Primary cortical folding in the human newborn: an early marker of later functional development. Brain 131, 2028-2041.

Fraser, W.I., King, K.M., Thomas, P., Kendell, R.E., 1986. The diagnosis of schizophrenia by language analysis. Br. J. Psychiatry 148, 275-278.

Glover, G.H., Li, T.Q., Ress, D., 2000. Image-based method for retrospective correction of physiological motion effects in fMRI: retroicor. Magn. Reson. Med. 44, 162-167.

Goghari, V.M., Sponheim, S.R., MacDonald, A.W., 2010. The functional neuroanatomy of symptom dimensions in schizophrenia: a qualitative and quantitative review of a persistent question. Neurosci. Biobehav. Rev. 34, 468-486 (3rd).

Goldman, H.H., Skodol, A.E., Lave, T.R., 1992. Revising axis V for DSM-IV: a review of measures of social functioning. Am. J. Psychiatry 149, 1148-1156.

Hoffman, R.E., Stopek, S., Andreasen, N.C., 1986. A comparative study of manic vs schizophrenic speech disorganization. Arch. Gen. Psychiatry 43, 831-838.

King, K., Fraser, W.I., Thomas, P., Kendell, R.E., 1990. Re-examination of the language of psychotic subjects. Br. J. Psychiatry 156, 211-215.

Kircher, T., Brohl, H., Meier, F., Engelen, J., 2018 Jun. Formal thought disorders: from phenomenology to neurobioleogy. Lancet Psychiatry 5 (6), 515-526.

Kirchner, W.K., 1958. Age differences in short-term retention of rapidly changing information. J. Exp. Psychol. 55, 352-358.

Lanczik, M., Keil, G., 1991. Carl Wernicke's localization theory and its significance for the development of scientific psychiatry. Hist Psychiatry 2, 171-180.

Leckman, J.F., Sholomskas, D., Thompson, W.D., Belanger, A., Weissman, M.M., 1982. Best estimate of lifetime psychiatric diagnosis: a methodological study. Arch. Gen. Psychiatry 39, 879-883.

Liddle, P.F., Ngan, E.T., Caissie, S.L., Anderson, C.M., Bates, A.T., Quested, D.J., White, R., Weg, R., 2002a. Thought and Language Index: an instrument for assessing thought and language in schizophrenia. Br. J. Psychiatry 181, 326-330.

Liddle, P.F., Ngan, E.T., Duffield, G., Kho, K., Warren, A.J., 2002b. Signs and symptoms of psychotic illness (SSPI): a rating scale. Br. J. Psychiatry 180, 45-50.

Mota, N.B., Vasconcelos, N.A., Lemos, N., Pieretti, A.C., Kinouchi, O., Cecchi, G.A., Copelli, M., Ribeiro, S., 2012. Speech graphs provide a quantitative measure of 
thought disorder in psychosis. PLoS ONE 7, e34928.

Mota, N.B., Furtado, R., Maia, P.P., Copelli, M., Ribeiro, S., 2014. Graph analysis of dream reports is especially informative about psychosis. Sci. Rep. 4, 3691.

Mota, N.B., Pinheiro, S., Sigman, M., Slezak, D.F., Cecchi, G., Copelli, M., Ribeiro, S., 2016a. The Ontogeny of Discourse Structure Mimics The Development of Literature. arXiv:1612.09268. arXiv Preprint.

Mota, N.B., Weissheimer, J., Madruga, B., Adamy, N., Bunge, S.A., Copelli, M., Ribeiro, S., 2016b. A Naturalistic Assessment of the Organization of Children's Memories Predicts Cognitive Functioning and Reading Ability. Mind, Brain, Education 10, 184-195.

Mota, N.B., Copelli, M., Ribeiro, S., 2017. Thought disorder measured as random speech structure classifies negative symptoms and schizophrenia diagnosis 6 months in advance. NPJ Schizophr. 3, 18.

Murray, A., 1943. Thematic Apperception Test. Harvard University Press, Cambridge, MA. US.

Nagels, A., Fahrmann, P., Stratmann, M., Ghazi, S., Schales, C., Frauenheim, M., Turner, L., Hornig, T., Katzev, M., Muller-Isberner, R., Grosvald, M., Krug, A., Kircher, T. 2016. Distinct neuropsychological correlates in positive and negative formal thought disorder syndromes: the thought and language disorder scale in endogenous psychoses. Neuropsychobiology 73, 139-147.

Palaniyappan, L., Liddle, P.F., 2012. Aberrant cortical gyrification in schizophrenia: a surface-based morphometry study. J. Psychiatry Neurosci. 37, 399-406.

Palaniyappan, L., Liddle, P.F., 2014. Diagnostic discontinuity in psychosis: a combined study of cortical gyrification and functional connectivity. Schizophr. Bull. 40, 675-684.

Palaniyappan, L., Al-Radaideh, A., Mougin, O., Gowland, P., Liddle, P.F., 2013a. Combined white matter imaging suggests myelination defects in visual processing regions in schizophrenia. Neuropsychopharmacology 38, 1808-1815.

Palaniyappan, L., Crow, T.J., Hough, M., Voets, N.L., Liddle, P.F., James, S., Winmill, L., James, A.C., 2013b. Gyrification of Broca's region is anomalously lateralized at onset of schizophrenia in adolescence and regresses at 2 year follow-up. Schizophr. Res. 147, 39-45.

Passby, L., Broome, M.R., 2017. Thought disorder. BJPsych Adv. 23, 321-323.
Perlini, C., Marini, A., Garzitto, M., Isola, M., Cerruti, S., Marinelli, V., Rambaldelli, G., Ferro, A., Tomelleri, L., Dusi, N., Bellani, M., Tansella, M., Fabbro, F., Brambilla, P., 2012. Linguistic production and syntactic comprehension in schizophrenia and bipolar disorder. Acta Psychiatr. Scand. 126, 363-376.

Posse, S., Wiese, S., Gembris, D., Mathiak, K., Kessler, C., Grosse-Ruyken, M.L., Elghahwagi, B., Richards, T., Dager, S.R., Kiselev, V.G., 1999. Enhancement of BOLDcontrast sensitivity by single-shot multi-echo functional MR imaging. Magn. Reson. Med. 42, 87-97.

Redpath, H.L., Cooper, D., Lawrie, S.M., 2013. Imaging symptoms and syndromes: similarities and differences between schizophrenia and bipolar disorder. Biol. Psychiatry 73, 495-496.

Schaer, M., Cuadra, M.B., Tamarit, L., Lazeyras, F., Eliez, S., Thiran, J.P., 2008. A surfacebased approach to quantify local cortical gyrification. IEEE Trans. Med. Imaging 27, 161-170.

Sommer, I.E., Derwort, A.M., Daalman, K., De Weijer, A.D., Liddle, P.F., Boks, M.P., 2010 Formal thought disorder in non-clinical individuals with auditory verbal hallucinations. Schizophr. Res. 118, 140-145.

Stephenson, K., Zelen, M., 1989. Rethinking centrality: methods and examples. Soc. Networks 11, 1-37.

Sumner, P.J., Bell, I.H., Rossell, S.L., 2018 Jan. A systematic review of the structural neuroimaging correlates of thought disorder. Neurosci. Biobehav. Rev. 84, 299-315.

Thomas, P., Kearney, G., Napier, E., Ellis, E., Leuder, I., Johnson, M., 1996. Speech and language in first onset psychosis differences between people with schizophrenia, mania, and controls. Br. J. Psychiatry 168, 337-343.

White, T., Hilgetag, C.C., 2011. Gyrification and neural connectivity in schizophrenia. Dev. Psychopathol. 23, 339-352.

WHO, 2003. Guidelines for ATC Classification and DDD Assignment, WHO Collaborating Centre for Drug Statistics and Methodology.

Xu, J.Q., Hui, C.L., Longenecker, J., Lee, E.H., Chang, W.C., Chan, S.K., Chen, E.Y., 2014 Executive function as predictors of persistent thought disorder in first-episode schizophrenia: a one-year follow-up study. Schizophr. Res. 159, 465-470. 